Nonlinear Processes in Geophysics (2003) 10: 13-26

Nonlinear Processes

in Geophysics

(C) European Geosciences Union 2003

\title{
Large amplitude solitary waves in and near the Earth's magnetosphere, magnetopause and bow shock: Polar and Cluster observations
}

\author{
C. Cattell ${ }^{1}$, C. Neiman ${ }^{1}$, J. Dombeck ${ }^{1}$, J. Crumley ${ }^{1}$, J. Wygant ${ }^{1}$, C. A. Kletzing ${ }^{2}$, W. K. Peterson ${ }^{3}$, F. S. Mozer ${ }^{4}$, and \\ M. André 5 \\ ${ }^{1}$ School of Physics and Astronomy, University of Minnesota, Minneapolis, MN 55455, USA \\ ${ }^{2}$ Dept. of Physics and Astronomy, University of Iowa, Iowa City, IA 52242, USA \\ ${ }^{3}$ Lockheed Martin Space Sciences Laboratory, Palo Alto, CA 94304, USA \\ ${ }^{4}$ Space Sciences Laboratory, University of California, Berkeley, CA 94720, USA \\ ${ }^{5}$ Swedish Institute for Space Physics, Uppsala, Sweden
}

Received: 29 November 2001 - Revised: 12 March 2002 - Accepted: 27 March 2002

\begin{abstract}
Solitary waves with large electric fields (up to 100 's of $\mathrm{mV} / \mathrm{m}$ ) have been observed throughout the magnetosphere and in the bow shock. We discuss observations by Polar at high altitudes $\left(\sim 4-8 R_{E}\right)$, during crossings of the plasma sheet boundary and cusp, and new measurements by Polar at the equatorial magnetopause and by Cluster near the bow shock, in the cusp and at the plasma sheet boundary. We describe the results of a statistical study of electron solitary waves observed by Polar at high altitudes. The mean solitary wave duration was $\sim 2 \mathrm{~ms}$. The waves have velocities from $\sim 1000 \mathrm{~km} / \mathrm{s}$ to $>2500 \mathrm{~km} / \mathrm{s}$. Observed scale sizes (parallel to the magnetic field) are on the order of $1-10 \lambda_{D}$, with $e \Phi / k T_{e}$ from $\sim 0.01$ to $O(1)$. The average speed of solitary waves at the plasma sheet boundary is faster than the average speed observed in the cusp and at cusp injections. The amplitude increases with both velocity and scale size. These observations are all consistent with the identification of the solitary waves as electron hole modes. We also report the discovery of solitary waves at the magnetopause, observed in Polar data obtained at the subsolar equatorial magnetopause. Both positive and negative potential structures have been observed with amplitudes up to $\sim 25 \mathrm{mV} / \mathrm{m}$. The velocities range from $150 \mathrm{~km} / \mathrm{s}$ to $>2500 \mathrm{~km} / \mathrm{s}$, with scale sizes the order of a kilometer (comparable to the Debye length). Initial observations of solitary waves by the four Cluster satellites are utilized to discuss the scale sizes and time variability of the regions where the solitary waves occur. Preliminary results from the four Cluster satellites have given a glimpse of the spatial and temporal variability of the occurrence of solitary waves and their association with other wave modes. In
\end{abstract}

Correspondence to: C. Cattell

(cattell@fields.space.umn.edu) all the events studied, significant differences were observed in the waveforms observed simultaneously at the four locations separated by $\sim 1000 \mathrm{~km}$. When solitary waves were seen at one satellite, they were usually also seen at the other satellites within an interval of a few seconds. In association with an energetic electron injection and a highly compressed magnetosphere, Cluster has observed the largest amplitude solitary waves $(>750 \mathrm{mV} / \mathrm{m}$ ) ever reported in the outer magnetosphere.

\section{Introduction}

Solitary waves have been identified throughout the Earth's magnetosphere at narrow boundaries, such as the plasma sheet boundary (Matsumoto et al., 1994; Franz et al., 1998; Cattell et al., 1999) and the bow shock (Bale et al., 1998; Mangeney et al., 1999), and in strong currents, such as those associated with auroral acceleration region (Temerin et al., 1982; Bostrom et al., 1988; Mozer et al., 1997; Ergun et al., 1998). They have also been seen at the high altitude polar cap boundary (Tsurutani et al., 1998), the high-latitude cusp boundary layer (Pickett et al., 1999), at high altitude cusp injections (Cattell et al., 2001b), and within the solar wind (Magenay et al., 1999). Recently Cattell et al. (2002) have reported the first observations of solitary waves at the magnetopause. Almost all of these observations have been of electron solitary waves, which are seen both at high and low altitudes and are observed for wide range of $f_{c e} / f_{p e}$. In contrast, to date, ion solitary waves have only been observed in the auroral zone at low altitudes in the region where $f_{c e} / f_{p e} \gg 1$. Recent studies of ion solitary waves in the auroral zone have been presented by Bounds et al. (1999), 
Crumley et al. (2001) and Dombeck et al. (2001). Solitary waves are usually observed as a symmetric bipolar pulse in the electric field component parallel to the geomagnetic field. Other shapes, such as three pulses (Mangenay et al., 1999) and offset bipolar pulses (Tsurutani et al., 1998), have also been described.

In this paper, we describe the results of a statistical study of large amplitude electron solitary waves observed at the plasma sheet boundary, and at the high altitude cusp and cusp injections using the Polar EFI instrument. We also present examples of solitary waves at the magnetopause obtained as the Polar orbit precessed into the subsolar magnetopause. In addition, we show initial examples of solitary waves from the four Cluster satellites in several different regions of the magnetosphere.

The electric field and spacecraft potential measurements utilized herein for the Polar studies were made by the double probe electric field instrument (Harvey et al., 1995). This instrument, which saturates at $\sim 1 \mathrm{~V} / \mathrm{m}$, obtains $3 \mathrm{D}$ measurements of the electric field in bursts (waveform capture) of high-time resolution data. Data obtained at two different burst rates (1600 and 8000 samples/s) are shown herein. The Polar high data rate bursts are not filtered. This was done to insure that the timing would be accurate (no frequency dependent phase shifts) and that the waveforms were not distorted by filtering. The low data rate bursts (not used for timing) have a 2-pole filter at $500 \mathrm{~Hz}$. The top frequency shown in all the Polar spectra is the Nyquist frequency. In addition to the electric field (potential difference between opposing probes), the spacecraft potential was utilized to indicate changes in density (Pedersen, 1995). The delay times between signals at opposing probes were examined using a cross-correlation analysis to estimate the propagation speed of electric field structures, utilizing an automatic program that first identifies bipolar pulses in the parallel component of the electric field data in the magnetic field-aligned coordinate system. The largest velocities which can be measured $(\sim 2500 \mathrm{~km} / \mathrm{s})$ correspond to the resolution of the time delay and depend on the boom orientation. Events where no time delay could be measured are included in the statistics as "infinite velocity". The automatic program used to calculate velocity also determines the structure width and solitary wave amplitude. Note that the method yields scale sizes which are approximately a factor of 4 larger than those obtained when the potential is fit to a Gaussian, since the duration of the solitary wave is based on the gradient in the parallel electric field changing its sign rather than on the Gaussian half-width. Details of this procedure and sample cross-correlation analysis are described elsewhere (Dombeck et al., 2001). Only the high rate data are utilized for timing and statistical studies. Note that wave amplitudes are given for the maximum electric field, not for the peak-to-peak amplitude. The AC magnetic field from the search coils (Gurnett et al., 1995) were sampled in the burst memory at the same rate as the electric field. DC magnetic field data, obtained from the fluxgate magnetometers (Russell et al., 1995) were utilized to determine the location of the boundaries. In addition, ion composition measurements made by the TIMAS instrument (Shelley et al., 1995) were examined to determine the probable source region and plasma characteristics. Hydra (Scudder et al., 1995) provided additional information on the particle distributions and moments in these regions.

The data for the Cluster study were obtained as the Cluster satellites traversed the bow shock, magnetopause, cusp and the plasma sheet boundary. The electric field and spacecraft potential measurements were made by the double probe electric field instruments (Gustaffson et al., 1988), which were designed to obtain bursts of high-time resolution data in many different modes. Electric field data at frequencies from DC to Nyquist frequency of $9 \mathrm{kHz}$ were obtained in the waveform captures described herein. The Cluster data shown are filtered with a 2-pole low pass filter at $4 \mathrm{kHz}$. The filters were designed so that there were no frequency-dependent phase shifts, again so that the timing and the shapes of the waveforms would be accurate. Spectra are shown up to $4 \mathrm{kHz}$. Single probe measurements were not transmitted for the events described herein, so solitary wave velocities could not be measured. The spacecraft potential was used to provide preliminary identification of the various boundaries and for determining boundary orientation and velocity. Note that the Cluster instruments only measure the electric field in the spin plane (approximately the ecliptic plane).

\section{Polar discovery of solitary waves at the magnetopause}

The precession of the Polar orbit so that apogee is in the equatorial plane has enabled a study of the subsolar, lowlatitude magnetopause with a modern suite of instruments. During March and April 2001, Polar repeatedly encountered the magnetopause. Cattell et al. (2002) reported the discovery of solitary waves in waveform capture data obtained at these magnetopause crossings. The three-dimensional nature of the Polar electric field instrument was critical for this discovery because the dominant component of the geomagnetic field is in the $Z$ direction (out of the ecliptic plane). Twodimensional instruments, which measure the component of the electric field in the $x-y$ (ecliptic) plane, would not usually be able to observe solitary waves.

An example of an equatorial magnetopause crossing on 2 May 2001 at 9.4 $R_{E}$ and 11.6 MLT, when the interplanetary magnetic field (IMF) was southward, is presented in Fig. 1. The transition from the magnetosphere (low density) to the magnetosheath (high density) can be clearly seen in the negative of the spacecraft potential (fifth panel) which is proportional to density. The transition is also clear in the magnetic field in geocentric solar magnetospheric (GSM) coordinates (panels 6-9). The electric field waveform capture was obtained throughout the magnetopause current layer, and part of the $\sim 34 \mathrm{~s}$ burst extended into the magnetosheath. The two perpendicular components in magnetic field-aligned coordinates (shown in panels 3 and 4) have very large amplitude waves (up to $\sim 150 \mathrm{mV} / \mathrm{m}$ ). In order to see the solitary wave signatures, it is necessary to look at the parallel com- 

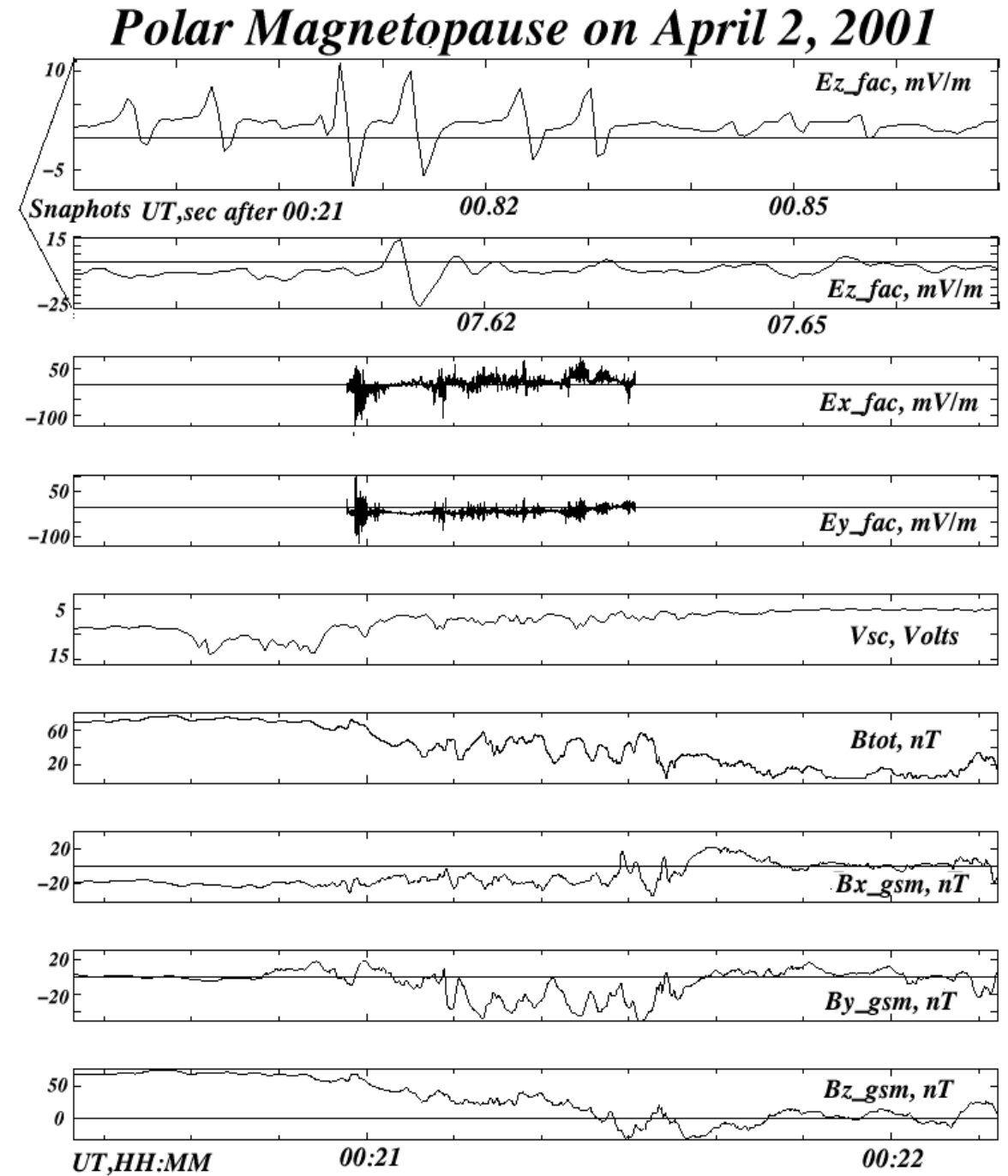

Fig. 1. A magnetopause crossing on 2 May 2001. From top to bottom, the panels are: two short snapshots of the field-aligned component of the electric field to show examples of the solitary waves; the two perpendicular components of the electric field in magnetic field-aligned system during the waveform capture; the negative of the spacecraft potential (proportional to density); and the magnitude of the magnetic field and the three components of the magnetic field in GSM. ponent for shorter intervals, as shown in the top two panels, which show two different $0.09 \mathrm{~s}$ intervals from the burst. The bipolar signature, which is typical of solitary waves, can be clearly seen. In the top panel, in addition to a train of spikes, several isolated solitary waves with amplitudes up to $12 \mathrm{mV} / \mathrm{m}$ can be seen. The second panel shows an isolated solitary wave with an amplitude of $\sim 25 \mathrm{mV} / \mathrm{m}$. The solitary wave speeds were too high for time delays to be observable in this low data rate (1600 samples/s) burst.

Figure 2 displays the $x, y$ and $z$ components of the electric field and the $x$ component of the search coil magnetic field data in field-aligned coordinates and their Fourier transforms. Most of the power in the perpendicular electric field is at frequencies of $<50 \mathrm{~Hz}$, consistent with lower hybrid waves, as has been previously observed (Treumann et al., 1995; Cattell et al., 1995). Note that Stasiewicz et al. (2001) suggested that the waves in this frequency range observed in the highlatitude magnetopause boundary layer were due to Doppler shifted kinetic Alfvén waves. The AC magnetic field has bursts of waves near the electron cyclotron frequency in the magnetic field minima. These waves can also be seen in the electric field when the larger amplitude low frequency electrostatic waves and the broadband signature (covering the full frequency range), due to the solitary waves, do not mask them. Observations of electron cyclotron waves at the magnetopause boundary layer have been described previously by Pickett et al. (2001).

Waveform captures were obtained near two magnetopause crossings on 23 March 2001 at $\sim 9 R_{E}$ and 12 MLT. The first waveform capture (from $\sim 01: 36: 14-01: 36: 19$ UT) occurred during a partial crossing of the magnetopause, just a few minutes prior to a complete outbound magnetopause crossing (at $\sim$ 01:39:10 UT) during southward IMF. Figure 3 shows the data from this event. The parallel electric field component for an interval of $0.4 \mathrm{~s}$ is plotted in the top panel, and the negative of the spacecraft potential and the magnetic field in the bottom five panels. At the time of the waveform capture, the density was increasing (as indicated by the spacecraft potential), and the $y$ and $z$ components and the magnitude of the magnetic field were decreasing, as expected if 

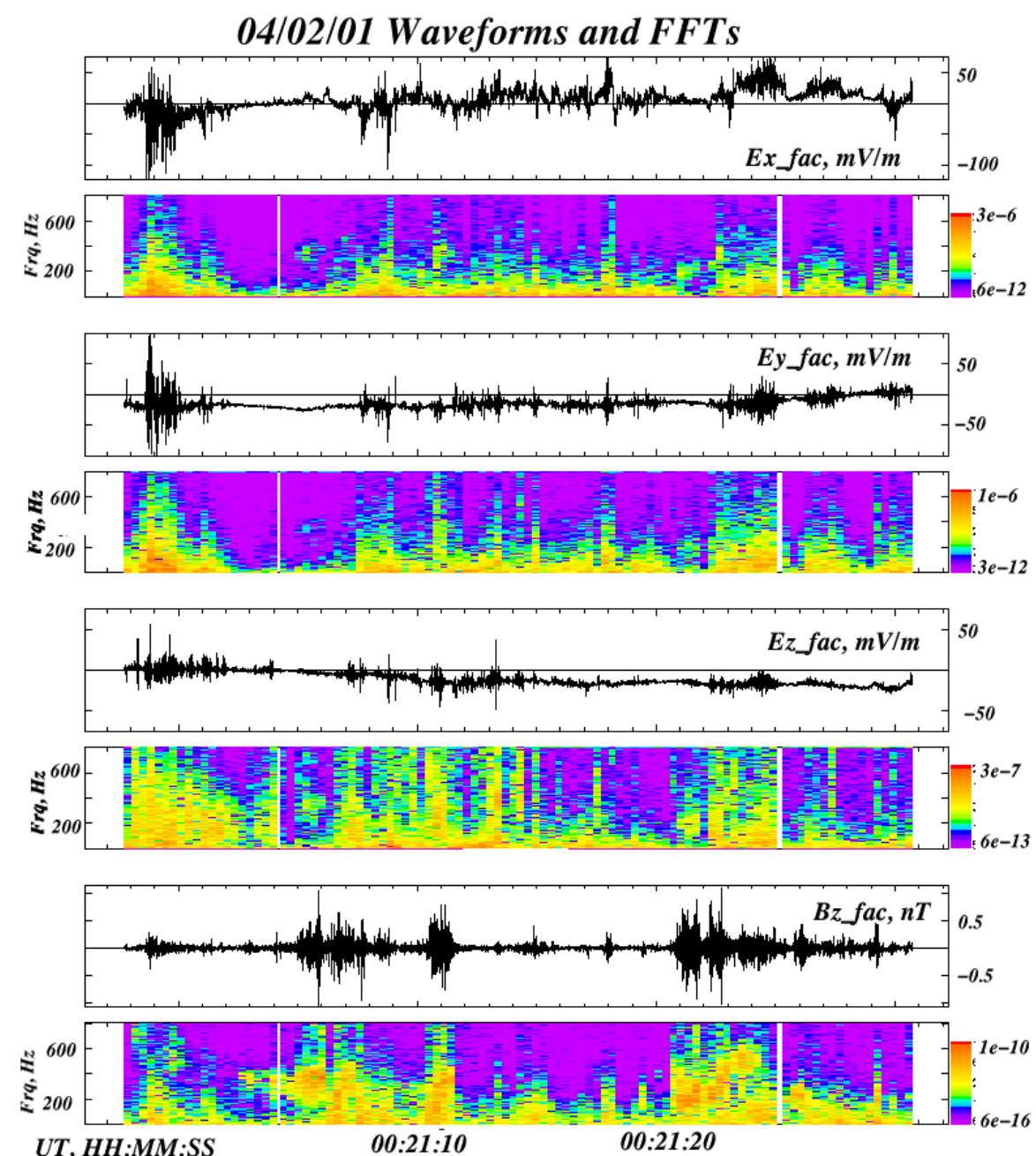

Fig. 2. The $x, y$ and $z$ components of the electric field in field-aligned coordinates and the $z$ component of the search coil magnetic field data, in fieldaligned coordinates, and their Fourier transforms from the waveform capture interval shown in Fig. 1. the satellite is moving from the magnetosphere towards the magnetosheath. The signatures of solitary waves with amplitudes of $\sim 5 \mathrm{mV} / \mathrm{m}$ can be seen in the top panel. The solitary waves had speeds of $\sim 800->2000 \mathrm{~km} / \mathrm{s}$. The second magnetopause event for which there was a waveform capture (shown in Fig. 4) occurred at a rapid crossing from the magnetosheath into the magnetosphere at 03:32 UT, followed by a crossing back into the magnetosheath at 03:33:20 UT. Solitary waves can be seen in the top panel, which shows a short interval during the waveform capture that was taken from 03:32:19-03:32:51 UT on the magnetospheric side of the magnetopause current layer. Note that some of the observed solitary waves have shapes other than a symmetric bipolar structure.

Additional examples are presented by Cattell et al. (2002), who also discussed the results of a study of all ten high-time resolution bursts obtained at and near the subsolar magnetopause. Solitary waves were observed in all except one. Solitary waves velocities from $\sim 150 \mathrm{~km} / \mathrm{s}$ to $>2000 \mathrm{~km} / \mathrm{s}$ and amplitudes of up to $25 \mathrm{mV} / \mathrm{m}$ were observed. The pulse duration ranges from $\sim 0.1 \mathrm{~ms}$ to $3 \mathrm{~ms}$, with a mean duration of $\sim 2 \mathrm{~ms}$. The scale sizes (parallel to the magnetic field) are the order of a kilometer, comparable to the Debye length for the usual range of plasma conditions at the magnetopause. Although most solitary waves were positive potential structures (consistent with the electron hole interpretation), there were also some negative potential structures. The fact that very low velocities and negative potential structures were sometimes observed suggests that there may be other types of solitary waves at the magnetopause. The wide range of velocities, however, is also consistent with the fact that mixtures of ionospheric, magnetosheath and magnetospheric particles occur in the magnetopause current layer.

\section{Polar statistical studies of electron solitary waves}

Observations of large amplitude solitary waves for a number of Polar crossings of the plasma sheet boundary, cusp and cusp particle injections at radial distances of $\sim 4-9 R_{E}$ have been presented by Cattell et al. (1999; 2001a; 2001b). These studies showed that the high altitude electron solitary waves have velocities from $\sim 1000 \mathrm{~km} / \mathrm{s}$ to $>2500 \mathrm{~km} / \mathrm{s}$ and amplitudes up to $200 \mathrm{mV} / \mathrm{m}$. A more detailed discussion of the relationship of the velocity to the particle characteristics can be 

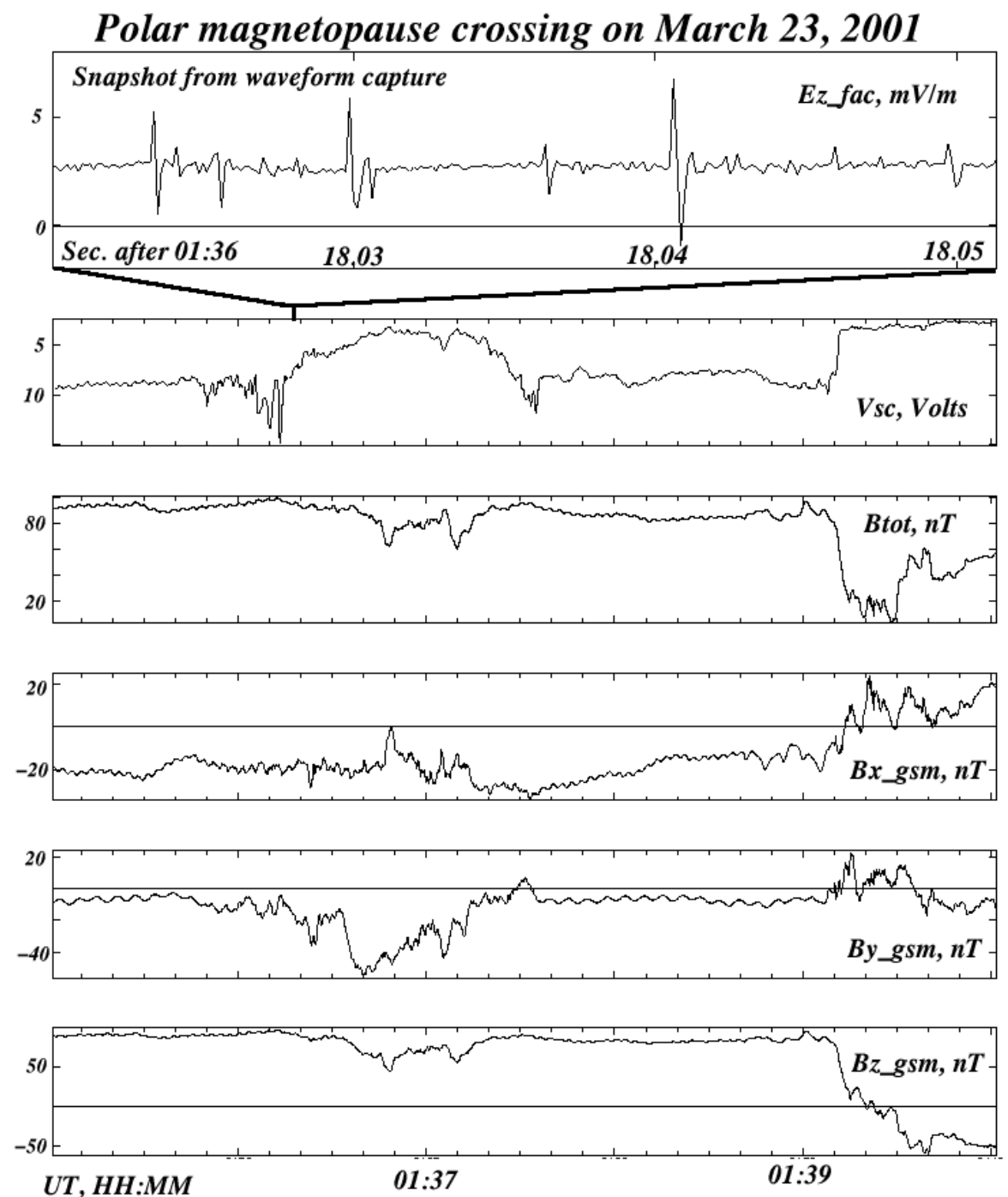

Fig. 3. A magnetopause crossing on 23 March 2001. From top to bottom, the panels are: an expanded view of the field-aligned component of the electric field for $0.12 \mathrm{~s}$ to show an example of the solitary waves; the negative of the spacecraft potential; the magnitude of the magnetic field and the three components of the magnetic field in GSM. found in these references. For the small set of events studied, the amplitude increased with both the velocity and the scale size, consistent with electron hole modes. The solitary waves were stable, based on the criterion developed by Muschietti et al. (1999). We discuss herein the results of a new Polar EFI statistical study of high altitude solitary waves.

All the high-time resolution (8000 samples/s) waveform capture intervals that were obtained at high altitudes during 1997 have been examined for solitary waves, utilizing the method of Dombeck et al. (2001). The ion composition data from TIMAS and the plasma data from Hydra were examined, in order to classify each interval as a plasma sheet boundary, cusp injection, cusp, or other region. A cusp injection event was defined as a case where the waveform capture was obtained at the leading edge of a velocity-dispersed cusp ion injection. This study was designed to provide statistical information on the characteristics of high altitude solitary waves and to determine whether these characteristics varied depending on the magnetospheric region.

Figure 5 presents a histogram of the solitary wave speed (and time delay) for the three spatial regions. Note that 'Inf' refers to cases which were moving so rapidly (usually $>2500 \mathrm{~km} / \mathrm{s}$ ) that no time delay could be measured. These cases are not included in the average. The average speed of solitary waves in the plasma sheet boundary was $\sim 1900 \mathrm{~km} / \mathrm{s}$, significantly larger than the average speed of solitary waves in the cusp and at cusp injections $(\sim 1400 \mathrm{~km} / \mathrm{s})$. The fact that solitary waves in the plasma sheet boundary propagate faster is even clearer when one examines the percentage of the solitary waves for which no time delay could be measured. Almost half were in the "Inf" category, whereas in the cusp injection and cusp event sets $<\sim 20 \%$ were moving too fast to be timed. This is consistent with the interpretation that the structures are electron holes and the fact that the electron temperature (and velocity) is lower in the cusp than in the plasma sheet boundary. 
Polar magnetopause crossing on March 23, 2001
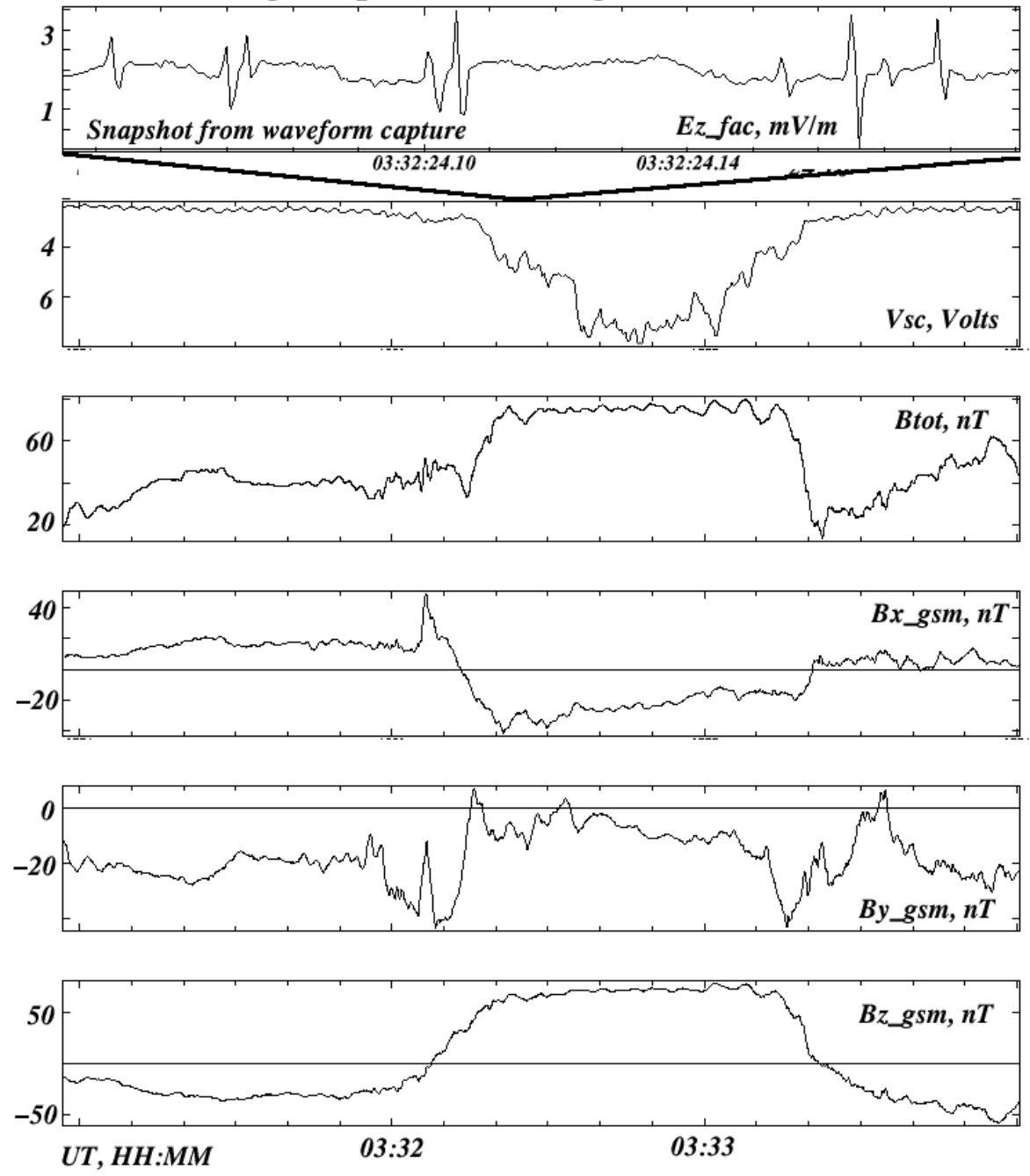

Fig. 4. Another magnetopause crossing on 23 March 2001. Same format as Fig. 3.
The solitary wave duration in each region had a mean of $\sim 2 \mathrm{~ms}$. The scale size normalized to the Debye length, determined using the Hydra key parameter electron density and temperature, were also examined for each region. The average width (parallel to the magnetic field) in the plasma sheet boundary is $\sim 1-2 \lambda_{D}$ and in the cusp (cusp injection), it is $\sim 5(8) \lambda_{D}$. This difference may not be significant because so many plasma sheet events had "infinite velocity"; therefore, only a minimum scale size could be computed. These widths are consistent with Goldman et al. (1999), who predicted scale sizes of $6-8 \lambda_{D}$. Note, however, that their simulations were for the strongly magnetized case, whereas the high altitude events are weakly magnetized.

A histogram of the amplitude of the solitary waves for all the high altitude events is shown in Fig. 6. Although most of the observed solitary waves have potentials of $<5 \mathrm{~V}$, there is a long tail with events out to amplitudes of $100 \mathrm{~V}$ (not shown). The average normalized amplitudes, $e \Phi / k T_{e}$, are of the order of 0.01 , with some events having amplitudes on the order of 1. The average amplitude was larger for the plasma sheet boundary events than for the cusp events, and very large amplitude waves were more common there. The relationship between potential amplitude and width, Fig. 7, shows a clear tendency for the solitary wave amplitude to increase with width. This relationship agrees with the prediction for a 1D BGK electron hole (Muschietti et al., 1999), but is opposite to the dependence predicted for a 1D, small amplitude acoustic soliton. Note that the amplitudes and scale sizes for the events with "Inf" velocity, plotted as asterisks, are lower bounds.

\section{Cluster observations of solitary waves and associated waves}

Preliminary studies of the Cluster waveform capture data have provided many examples of solitary waves and other nonlinear waveforms near the bow shock, at the plasma sheet 

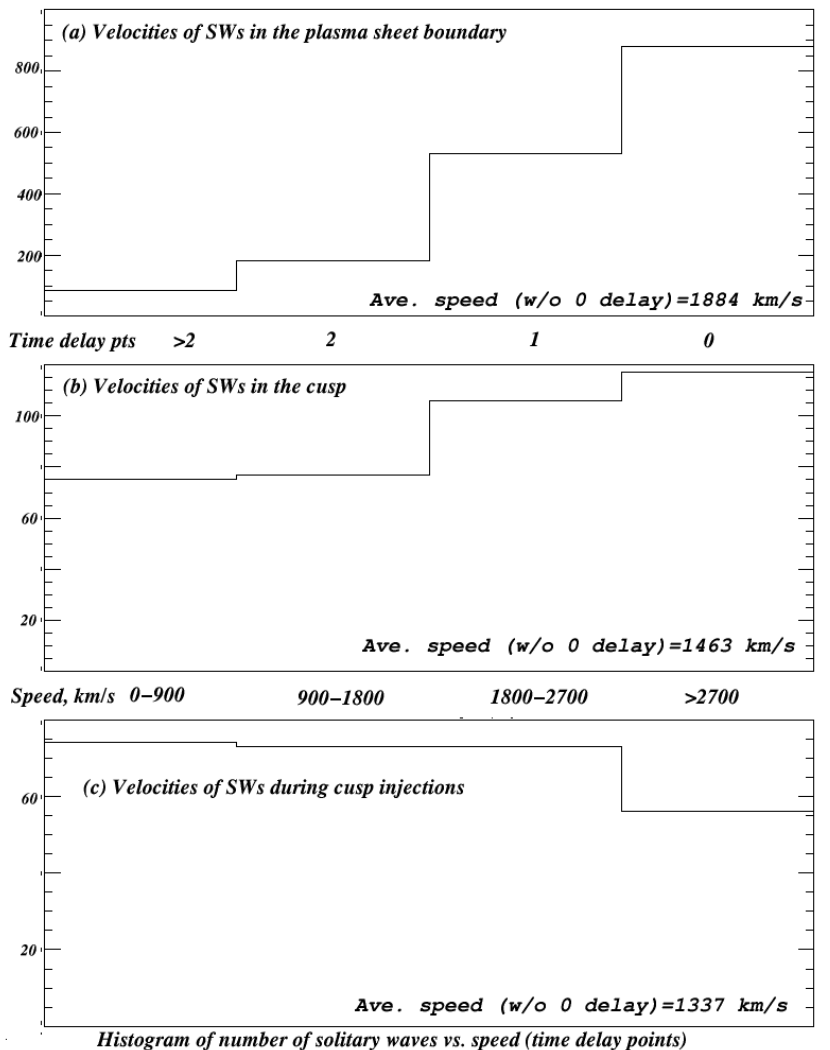

Fig. 5. A histogram of the solitary wave speed for the three spatial regions. Note that "Inf" refers to cases which were moving so rapidly (usually $>2500 \mathrm{~km} / \mathrm{s}$ ) that no time delay could be measured.

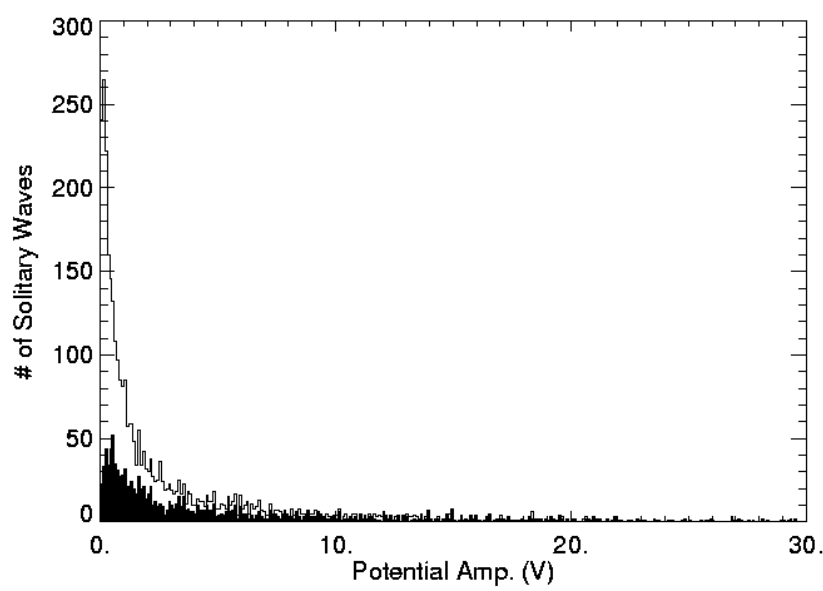

Fig. 6. A histogram of the amplitude of the solitary waves observed during all the high altitude, high data rate bursts during 1997. Events plotted in black correspond to the 'Inf' cases and represent a minimum potential.

boundary, in the cusp and during high altitude auroral zone crossings. The Cluster data provide the opportunity to determine where, within a boundary or current sheet, solitary waves occur, the time and spatial variability of the occur-
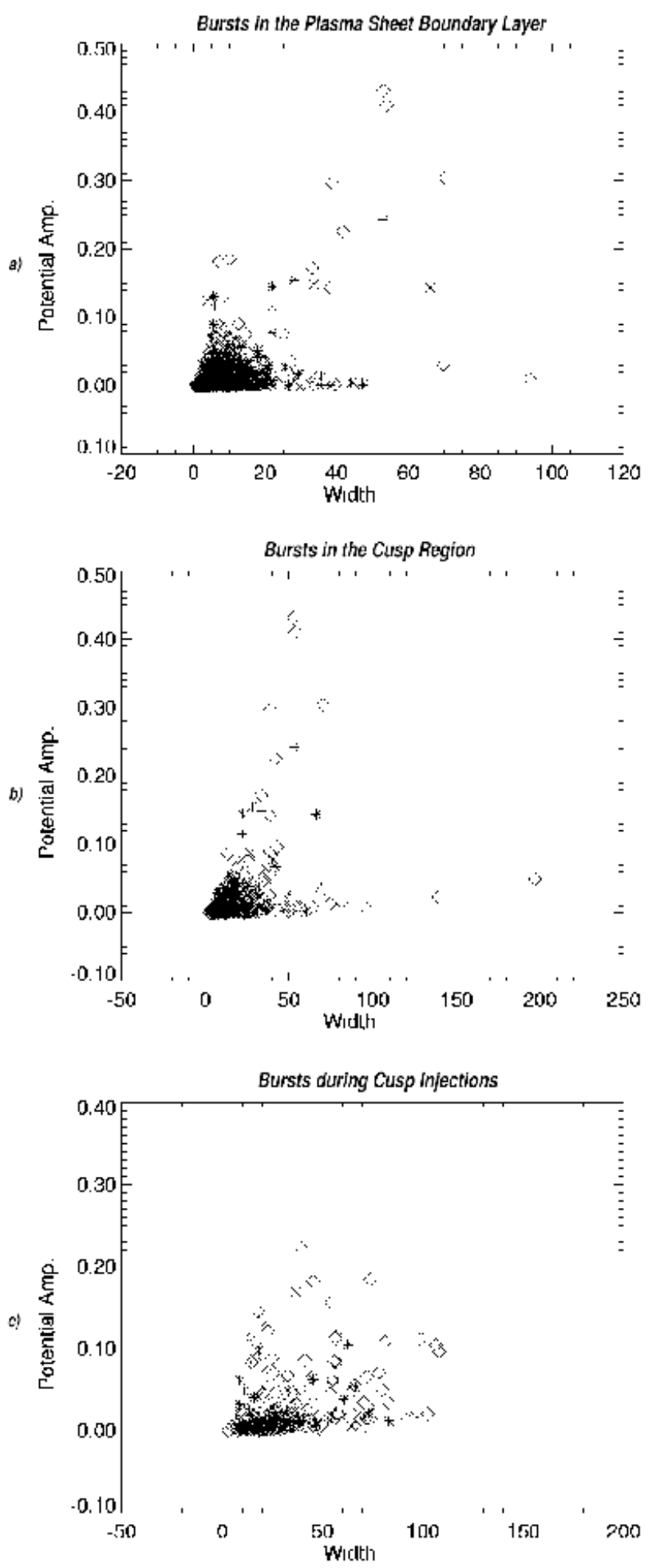

Fig. 7. A scatter plot of amplitude versus width for all the high altitude solitary wave events. The amplitudes and scale sizes for the 'Inf' cases, plotted as asterisks, are lower bounds. The increase in amplitude with scale size is consistent with a BGK electron hole.

rence and properties of solitary waves, and their relationship to other types of waves and wave packets. Examples taken 
on three days at different locations are presented below.

On 7 March 2001, three of the Cluster satellites collected waveform captures at $\sim 8.2 R_{E}$ and $\sim 9$ MLT at the outer edge of the cusp. An overview of the complete waveform capture for one component of the electric field and its Fourier transform from $\mathrm{SC} 2, \mathrm{SC} 3$, and $\mathrm{SC} 4$ is shown in Fig. 8a. Note that the scale for the electric field is $\pm 10 \mathrm{mVm}$ for $\mathrm{SC} 2$ and $\mathrm{SC} 4$, and $\pm 40 \mathrm{mV} / \mathrm{m}$ for SC3. The scales for the power spectra are the same for all three satellites. At this time, SC3 was closest to the equatorial plane and SC4 was the farthest from the equatorial plane. It is clear that the character of the waves is quite different on the 3 satellites. The waves observed on SC 3 had the largest amplitude (up to $40 \mathrm{mV} / \mathrm{m}$ ) and were the most spiky, including numerous solitary waves. Regions containing solitary waves can be identified in the spectra as broadband 'lines' from $0-\sim 4 \mathrm{kHz}$. Although all three satellites observed waves at frequencies of $\sim 2-3 \mathrm{kHz}$, and harmonics, these waves were the strongest on SC4. These frequencies are close to both the electron cyclotron frequency $\left(f_{c e} \sim 2.8 \mathrm{kHz}\right)$ and the electron plasma frequency $\left(f_{p e} \sim 2-4 \mathrm{kHz}\right.$ ), which is typical for this region. The harmonics observed in the spectra may be due to nonlinear steepening of the waveforms, as discussed by Temerin et al. (1979) for electrostatic ion cyclotron waves. Note that electron cyclotron waves have been observed at the highlatitude magnetopause boundary layer (Pickett et al., 2001). Several snapshots from the interval (Fig. 8b-d) show the highly variable character of the waves. The $\sim 0.1 \mathrm{~s}$ interval in Fig. 8b was taken when the waves on SC2 had a very small amplitude $(<1 \mathrm{mV} / \mathrm{m})$, and the solitary waves were observed by both SC3 (up to $\sim 10 \mathrm{mV} / \mathrm{m}$ ) and SC4 (up to $\sim 4 \mathrm{mV} / \mathrm{m}$ ). In addition, SC4 observed quasi-monochromatic waves at 2$2.5 \mathrm{kHz}$. The $\sim 0.05 \mathrm{~s}$ interval in Fig. $8 \mathrm{c}$, which occurred when data from SC4 were not available, has examples of solitary waves from both $\mathrm{SC} 2$ and $\mathrm{SC} 3$. Note that the solitary wave on SC2 had a much lower amplitude, consistent with the fact that the electric field perturbations on SC2 were small compared to the other satellites throughout the waveform capture. Figure $8 \mathrm{~d}$ shows a period where the waves on both SC2 and SC4 had a small amplitude, and the solitary waves, with amplitudes up to $\sim 30 \mathrm{mV} / \mathrm{m}$, were seen on SC3. During this interval, there were no structures observed in the spacecraft potential (density) that could be clearly identified at all four satellites to use for timing.

On 3 March 2001, the Cluster satellites were at $\sim 15 R_{E}$ near noon, close to the bow shock, and all four obtained waveform captures. Figure 9a shows an overview of the observations with one component of the electric field and its Fourier transform from each satellite. All four spacecraft see intense, bursty wave packets with amplitudes up to $\sim 200 \mathrm{mV} / \mathrm{m}$. SC 1 , which is the farthest from the equatorial plane, sees more continuous wave packets than the other spacecraft. Wave power maximizes between $\sim 2-4 \mathrm{kHz}$ throughout much of this interval. In this region, $f_{p e}$ typically ranges from $\sim 3-9 \mathrm{kHz}$ and $f_{c e}$ from $\sim 200-300 \mathrm{~Hz}$. Figure $9 \mathrm{~b}$ shows data from an $\sim 0.6 \mathrm{~s}$ period. The highly variable modulation of the waves suggests complex nonlin- ear interactions (such as the modulational instability), which vary over the $\sim 400-900 \mathrm{~km}$ separations of the satellites. In an $\sim 0.02 \mathrm{~s}$ interval shown in Fig. 9c, all 4 satellites observe wave packets with comparable amplitudes and frequencies. SC3 observes a solitary structure at $\sim 07: 17: 05.885$ UT. The observed waves are in the range, $f_{p i}<f<f_{p e}$, consistent with ion acoustic waves, although it is also possible that the very narrow wave packets are Langmuir waves. Mangeney et al. (1999) have discussed observations of narrow modulated bursts in both the ion acoustic and Langmuir frequency ranges, using Wind data in the solar wind. Observations of intense waves at the plasma frequency in the electron foreshock have been discussed by Bale et al. (2000). Additional analysis will be required to definitely identify the wave mode or modes observed by Cluster.

There were also large scale structures in the spacecraft potential in the interval around the time of the waveform captures obtained on 3 March 2001. The timing of several discontinuities seen in the spacecraft potential indicated that these large-scale structures were moving at speeds of $\sim 300 \mathrm{~km} / \mathrm{s}$, primarily anti-sunward, with a small duskward component. The structures were observed first by SC2 and $\mathrm{SC} 3$, then SC4, and finally SC1.

Cluster encountered the high altitude auroral zone (plasma sheet) at a radial distance of $\sim 4.2 R_{E}$ at 21:30 MLT and $\sim 62^{\circ}$ ILAT (south) on 31 March 2001 at $\sim 06: 44$ UT. The magnetosphere was highly compressed at this time, and the RAPID instrument observed an intense injection of electrons $\sim 14$ min earlier (Baker et al., 2001). Figure 10 shows one component of the waveform capture electric field data from the four satellites, indicating that not only all observed large amplitude waves but also the types of waves are quite variable. Figure 11 plots an $\sim 0.03 \mathrm{~s}$ interval from each satellite, to illustrate that solitary waves were detected at all four locations, with amplitudes often reaching $500-750 \mathrm{mV} / \mathrm{m}$. It can be seen that, at times, the amplitude is saturated, indicating that amplitudes sometimes were larger than $750 \mathrm{mV} / \mathrm{m}$. These are much larger than the previously reported largest solitary waves at high altitudes (Cattell et al., 2001a), which were $200 \mathrm{mV} / \mathrm{m}$ and observed by Polar at $\sim 5 R_{E}$. Although the solitary waves are usually distinct individual pulses, they sometimes occur in wave trains (as near 06:44:01.5 on SC3). As discussed in relationship to previous examples on both Polar and Cluster, although symmetric bipolar pulses are the predominant signatures, more complex shapes are also seen, as has been reported previously.

\section{Discussion and conclusions}

We have described three studies of solitary waves which are most commonly observed as bipolar pulses in the electric field parallel to the background magnetic field and which are ubiquitous in boundaries and other regions with strong currents and/or electron beams. The Polar observations at the subsolar equatorial magnetopause have provided the first evidence for the occurrence of solitary waves in and near 

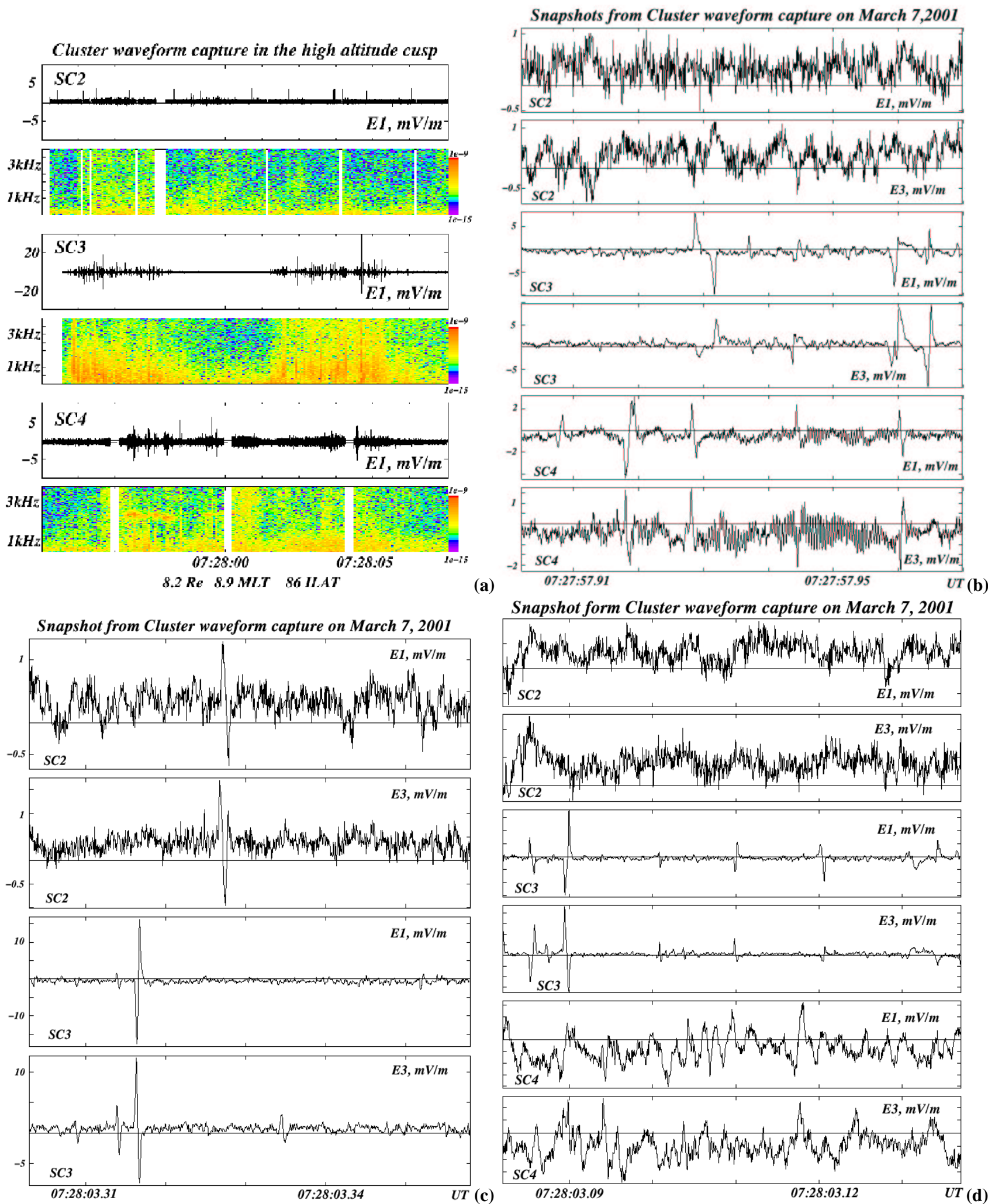

Fig. 8. Solitary waves and associated wave packets on 3 Cluster satellites on 7 March 2001 at $\sim 8.2 R_{E}$ and $\sim 9$ MLT in the region of the cusp. (a) Overview of the complete waveform capture with 1 component of the electric field from SC2, SC3, and SC4 and the Fourier spectra; (b), (c) and (d) Shorter snapshots within the burst, showing solitary waves. Note that the scale for the electric field is $\pm 10 \mathrm{mVm}$ for $\mathrm{SC} 2$ and SC4, and $\pm 40 \mathrm{mV} / \mathrm{m}$ for SC3. The scales for the power spectra are the same for all three satellites. 
Cluster waveform capture near bowshock on March 3, 2001
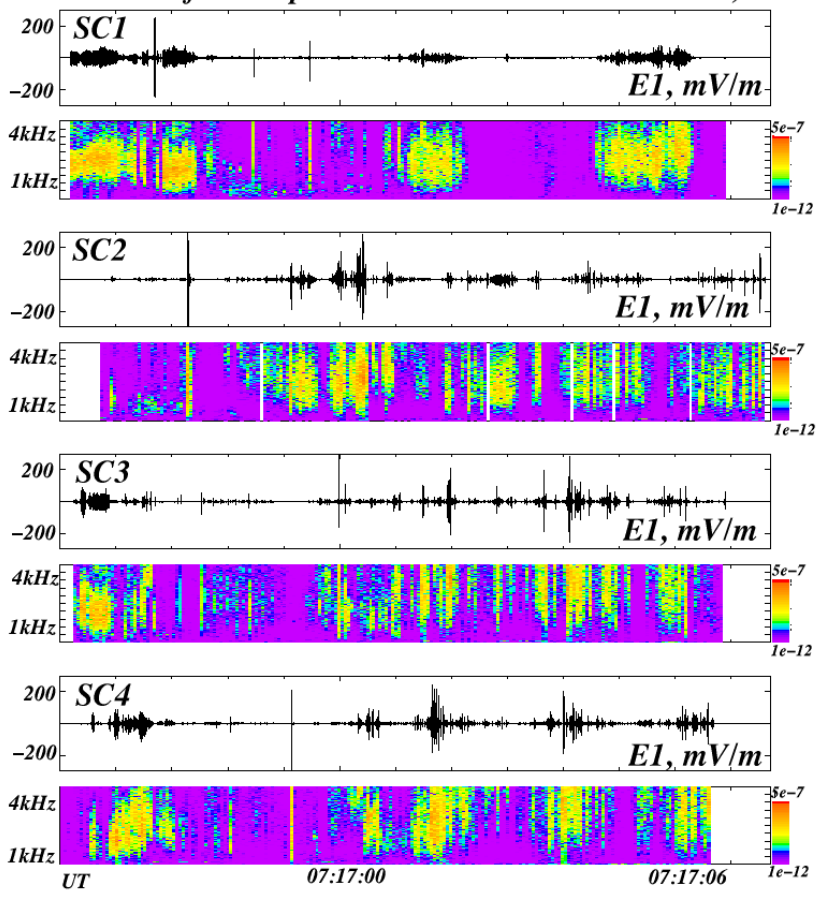

$(12,3,9) \mathrm{Re} \quad 15.5 \mathrm{Re} \quad 12.1 \mathrm{MLT}$
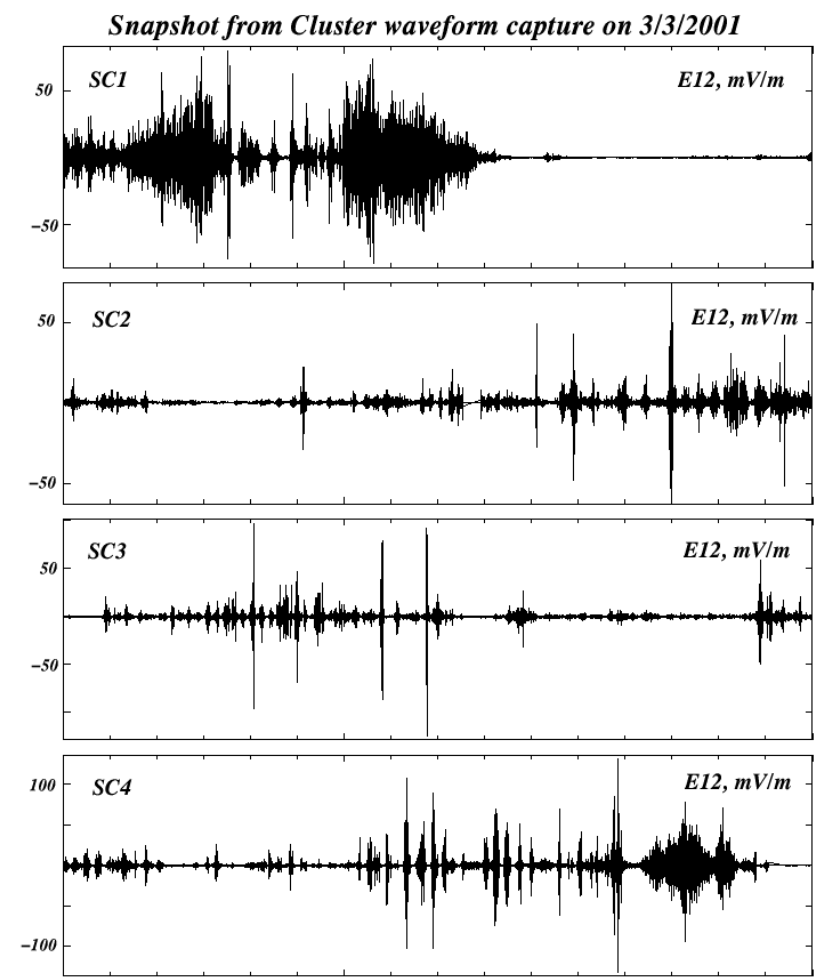

(a)

17:07:06 UT Snapshot duration - 0.598s

(b)

Snapshots from Cluster waveform capture on 3/3/2001

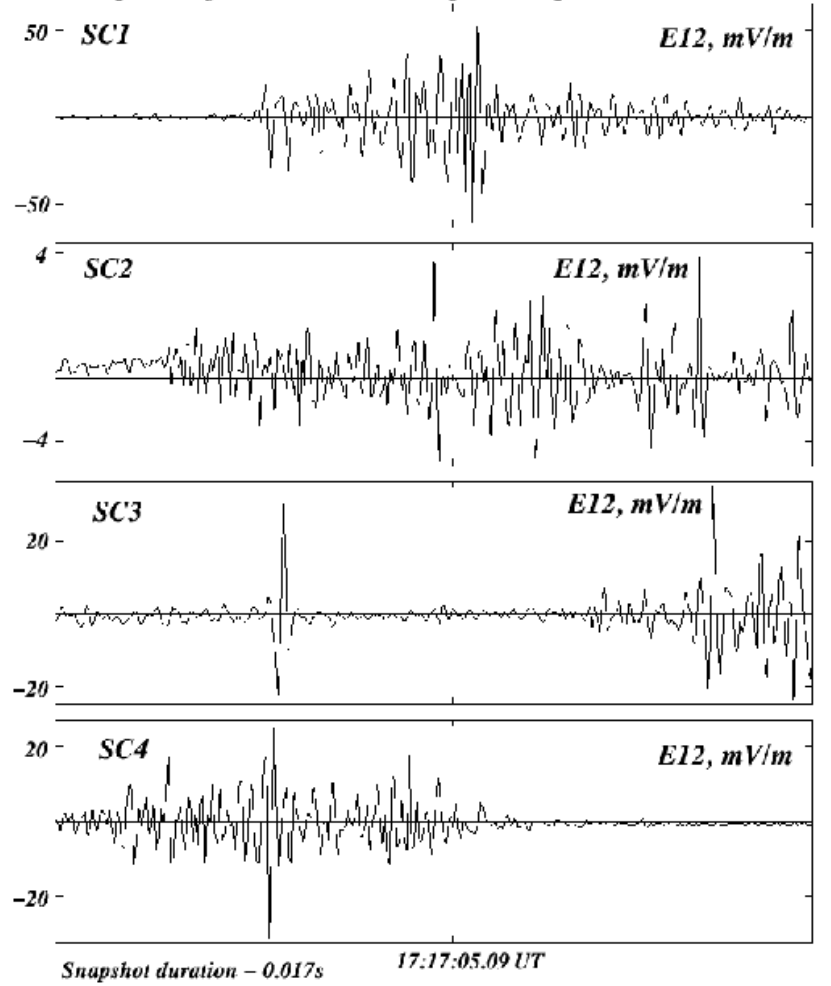

(c)

Fig. 9. Waveform capture obtained on 3 March 2001 at $\sim(12,3,9) R_{E}$ near noon, close to the bow shock: (a) Overview of the complete waveform capture with 1 component of the electric field from SC1, SC2, SC3, and SC4 and the Fourier spectra; (b) and (c) shorter snapshots. 


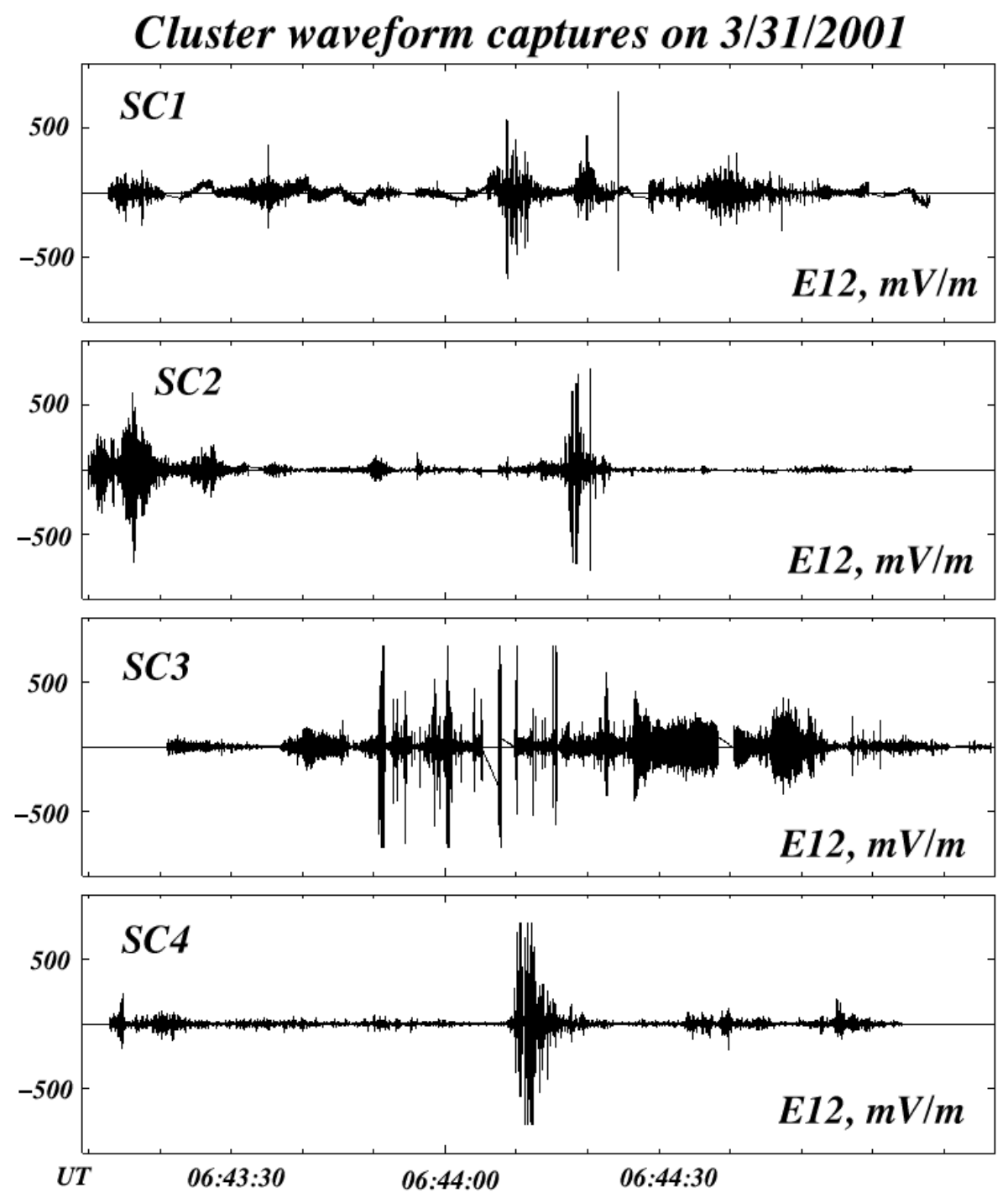

Fig. 10. One component of the waveform capture electric field data from the four satellites on 31 March 2001 near the plasma sheet boundary. the magnetopause current layer. Solitary waves at the magnetopause occur both as individual spikes and in trains of spikes, with amplitudes up to $\sim 25 \mathrm{mV} / \mathrm{m}$, an average duration of $\sim 2 \mathrm{~ms}$, scale sizes of the order of a kilometer (comparable to the Debye length) and velocities from $\sim 150 \mathrm{~km} / \mathrm{s}$ to $>2000 \mathrm{~km} / \mathrm{s}$. They are often associated with very large amplitude waves in either or both the electric and magnetic fields. Although most of the observed signatures are consistent with an electron hole mode, the events with very low velocities and the few negative potential structures may be indicative of a second type of solitary wave in the magnetopause current layer. Simulations have shown that electron holes interact strongly with electrons (Omura et al., 1996; Goldman et al., 1999; and references therein); therefore, the solitary waves may be an important source of dissipation and diffusion at the magnetopause.

The statistical study described herein of high altitude solitary waves observed by the Polar EFI provided information on the differences between properties of solitary waves at the plasma sheet boundary, cusp injections and the cusp. No significant differences were observed between the properties within the cusp and at cusp injections. The average speed for the plasma sheet boundary events was significantly faster than the average speed in the cusp events. The statistical results on the scale sizes and potentials were limited by the fact that, for events with zero time delay, only a lower limit on the potential and scale size could be obtained. Given this limitation, the observations are consistent with the conjecture that the scale sizes of the solitary waves are of the order of 110 Debye lengths in all regions, and that the potentials were larger in the plasma sheet boundary. The mean solitary wave duration was $\sim 2 \mathrm{~ms}$ in all three regions. We did not address the shape of the solitary waves. Franz et al. (2000) described the results of a statistical study of solitary waves observed at high altitude on Polar by the PWI instrument. They concluded that the ratio of parallel to perpendicular scale size 

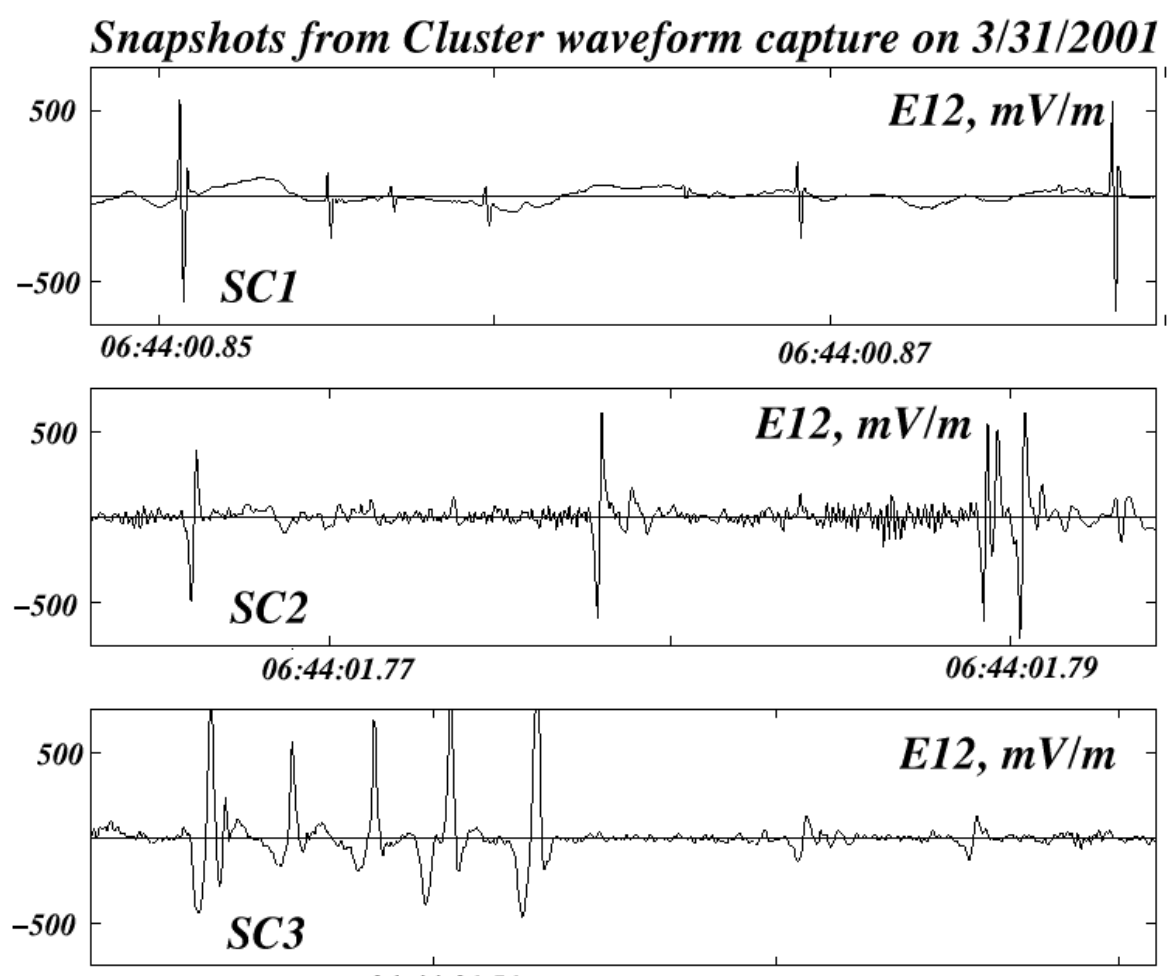

06:44:01.51

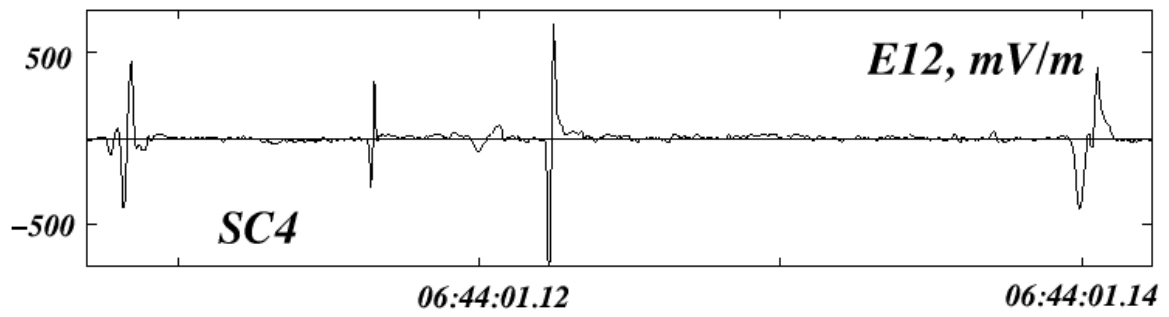

Note: Times of snapshots are different.
Fig. 11. An approximately $0.03 \mathrm{~s}$ interval for each Cluster satellite, from the waveform capture in Fig. 10. (Note that each interval is at a different time.) depended on $\left(f_{c e} / f_{p e}\right)$, and with the perpendicular scale size increasing with respect to the parallel scale size as $\left(f_{c e} / f_{p e}\right)$ became less than one.

Since all previous solitary wave observations have been made by a single spacecraft, it has not been possible to determine the spatial and temporal relationship between regions within boundaries and/or specific free energy sources and the occurrence of solitary waves. In this preliminary analysis, it has not been possible to fully exploit the resources of the Cluster mission, for example, to determine the relationship between the free energy source and the wave modes seen at the four different locations. Preliminary results from the four Cluster satellites have, however, given a glimpse of the spatial and temporal variability of the occurrence of solitary waves and their association with other wave modes. In all the events studied, significant differences were observed in the types of waveforms observed simultaneously at four locations separated by $\leq 1000 \mathrm{~km}$. However, when solitary waves are seen at one satellite, they are usually also seen at the other satellites within an interval of a few seconds. The cusp event had the most variability in typical amplitudes, with the largest amplitudes at SC3, which was the closest to the equatorial plane. In contrast, the event obtained near the bow shock at noon had the least variability between the four spacecraft in the typical wave amplitudes. The characteristics of the waveforms were also very similar on all spacecraft, with the exception of SC1, which saw more continuous waves. Note that $\mathrm{SC} 1$ was the farthest from the equatorial plane and the timing of large-scale density structures near the time of the burst showed that these structures encountered SC1 last. The delay was $<2-3 \mathrm{~s}$ and the observed differences are not consistent with the idea that the observed waves are convected past the satellites with the large-scale structures. Cluster has observed the largest amplitude solitary waves $(750 \mathrm{mV} / \mathrm{m})$ in the outer magnetosphere. These solitary waves, seen at all 4 spacecraft, occurred at $\sim 4.2 R_{E}$ 
within the plasma sheet on auroral field lines, in association with a highly compressed magnetosphere.

Acknowledgements. Analysis of Polar electric and magnetic field data was supported by the NASA under grants NAG 5-3182, NAG5-3217, and NAG5-7885. J. Dombeck was supported by NASA GSRP NGT5-50251 and J. Crumley by NGT5-50293. Analysis of the Cluster electric field data was supported by NAG59985. Analysis of HYDRA data at University of Iowa was performed under NASA grant number NAG5-2231 and DARA grant 50 OC 89110 . We would also like to thank C. T. Russell for use of the fluxgate magnetometer data and J. Vernetti for work on the Cluster burst-mode software. C. Cattell is a Cotrell Scholar. Her participation in this workshop was supported by Research Corporation.

\section{References}

Bale, S., Kellogg, P., Larson, D., et al.: Bipolar electrostatic structures in the shock transition region: Evidence of electron phase space holes, Geophys. Res. Lett., 25, 2929, 1998.

Bale, S., Larson, D., Lin, A., et al.: On the beam speed and wave number of intense electron plasma waves near the foreshock edge, J. Geophys. Res., 105, 27 353, 2000.

Baker, D., Ergun, R. E., Burch, J., et al.: A Telescopic and Microscopic View of a Magnetospheric Substorm on 31 March 2001, Geophys. Res. Lett., 10.1029/2001/GL014491, 2002.

Bostrom, R., Gustafsson, G., Hollback, B., et al.: Characteristics of solitary waves and double layers in the magnetospheric plasma, Phys. Rev. Lett., 61, 82, 1988.

Bounds, S., Pfaff, R., Knowlton, A., et al.: Solitary potential structures associated with ion and electron beams near $1 R_{E}$, J. Geophys. Res., 104, $28709,1999$.

Cattell, C., Wygant, J., Mozer, F., Okada, T., Kokubun, S., and Yamamoto, T.: ISEE-1 and Geotail observations of low frequency waves at the magnetopause, J. Geophys. Res., 100, 11 823, 1995.

Cattell, C., Wygant, J., Dombecks, J., et al.: Observations of large amplitude parallel electric field wave packets at the plasma sheet boundary, Geophys. Res. Lett., 25, 857-900, 1998.

Cattell, C., Dombecks, J., Wygant, J., et al.: Comparisons of Polar satellite observations of solitary wave velocities in the plasma sheet boundary and the high altitude cusp to those in the auroral zone, Geophys. Res. Lett., 26, 425-428, 1999.

Cattell, C., Dombecks, J., Keiling, A., et al.: Comparison of solitary waves and wave packets observed at plasma sheet boundary to results from the auroral zone, Phys. Chem. Earth, 26, 97, 2001 a.

Cattell, C., Crumley, J., Dombeck, J., Lysak, R., Kletzing, C., Peterson, W. K., and Collin, H.: Polar Observations Of Solitary Waves At High And Low Altitudes And Comparison To Theory, Adv. Space Res., 28, 1631, 2001b.

Cattell, C., Crumley, J., Dombeck, J., Wygant, J., and Mozer, F. S.: Polar observations of solitary waves at the Earth's magnetopause, Geophys. Res. Lett., 29, 2001GL01400469-1, 2002.

Crumley, J., Cattell, C., Lysak, R., and Dombeck, J.: Studies of ion solitary waves using simulations including hydrogen and oxygen beams, J. Geophys. Res., 106, 6007, 2001.

Dombeck, J., Cattell, C., Crumley, J., Peterson, W., Collin, H., and Kletzing, C.: Observed trends in auroral zone ion solitary wave structure characteristics using data from Polar, J. Geophys. Res., 106, $19013,2001$.
Ergun, R., Carlson, C., McFadden, J., et al.: FAST satellite observations of large amplitude solitary wave structures, Geophys. Res. Lett., 25, 2041-2044, 1998.

Franz, J., Kintner, P., and Pickett, J.: Polar observations of coherent electric field structures, Geophys. Res. Lett., 25, 1277-1280, 1998.

Franz, J., Kintner, P., and Pickett, J.: On the perpendicular scale size of electron phase space holes, Geophys. Res. Lett., 27, 169-172, 2000.

Harvey, P., Mozer, F., Pankow, D., et al.: The Electric Field Instrument on the Polar Satellite, in: The Global Geospace Mission, (Ed) Russell, C. T., p. 583, 1995.

Goldman, M., Oppenheim, M., and Newman, D.: Nonlinear twostream instabilities as an explanation for auroral biploar wave structures, Geophys. Res. Lett., 26, 1821-1824, 1999.

Gurnett, D., Persoon, A., Randall, R., et al.: The Polar Plasma Wave Experiment, in: The Global Geospace Mission, (Ed) Russell, C. T., p. 597, 1995 .

Gustafsson, G., Aggson, T., Bostrom, R., et al.: The spherical probe electric field and wave experiment, in: The Cluster MissionScientific and Technical Aspects of the Instruments, ESA SP1103, ESA Publications Div., Noordwijk, The Netherlands, p. $31,1988$.

Mangeney, A., Salem, C., Lacombeet, C., et al.: WIND observations of coherent electrostatic waves in the solar wind, Ann. Geophysicae, 17, 307, 1999.

Matsumoto, H., Kojima, H., Miyatake, T., et al.: Electrostatic solitary waves (ESW) in the magnetotail: BEN waveforms observed by Geotail, Geophys. Res. Lett., 21, 2915, 1994.

Matsumoto, H., Frank, L., Omura, Y., et al.: Generation mechanism of ESW based on the GEOTAIL Plasma Wave Observation, Plasma Observation and Particle Simulation, Geophys. Res. Lett., 26, 421-424, 1999.

Mottez, F., Perrault, A., Roux, A., et al.: Coherent structures in the magnetotail triggered by counterstreaming electron beams, J. Geophys. Res., 102, 11 399, 1997.

Mozer, F., Ergun, R., Temerin, M., et al.: New features in time domain electric field structures in the auroral acceleration region, Phys. Rev. Lett., 79, 1281, 1997.

Muschietti, L., Ergun, R., Roth, I., et al.: Phase-space electron holes along magnetic field lines, Geophys. Res. Lett., 26, 1093, 1999.

Omura, Y., Matsumoto, H., Miyake, T., et al.: Electron beam instabilities as the generation mechanism of electrostatic solitary waves in the magnetotail, J. Geophys. Res, 101, 2685-2697, 1996.

Pedersen, A.: Solar wind and magnetospheric plasma diagnostics by spacecraft electrostatic potential measurements, Ann. Geophysicae, 13, 118, 1995.

Pickett, J. S., Gurnett, D., Menietti, A., et al.: Plasma waves observed during cusp energetic particle events and their correlation with Polar and Akebono satellite and ground data, Adv. Space Res., 24, 23, 1999.

Pickett, J. S., Franz, J., et al.: Plasma waves observed in the cusp turbulent boundary layer: An analysis of high time resolution wave and particle measurements from the Polar spacecraft, J. Geophys. Res, 106, 19 081, 2001.

Russell, C. T., Share, R., Means, J., et al.: The GGS/Polar Magnetic Fields Investigation, in: The Global Geospace Mission, (Ed) Russell, C. T., p. 563, 1995.

Scudder, J., Hunsacker, F., Miller, G., et al.: Hydra-A 3-D electron and ion hot plasma instrument for the Polar spacecraft of the GGS mission, in: The Global Geospace Mission, (Ed) Russell, 
C. T., p. 459, 1995.

Shelley, E. G., Ghelmetti, A., Balsinger, H., et al.: The Toroidal Imaging Mass-Angle Spectrograph (TIMAS) for the Polar Mission, in: The Global Geospace Mission, (Ed) Russell, C. T., p. 497, 1995.

Stasiewicz, K., Seyler, C., Mozer, F., Gustaffson, G., Pickett, J., and Popielawska, B.: Magnetic bubbles and kinetic Alfven waves in the high-latitude magnetopause boundary, J. Geophys. Res., 106, 29503, 2001.

Temerin, M., Woldorff, M., and Mozer, F. S.: Nonlinear steepening of the electrostatic ion cyclotron wave, Phys. Rev. Lett., 43, 1941, 1979.
Temerin, M., Cerny, K., Lotko, W., et al.: Observations of solitary waves and double layers in the auroral plasma, Phys. Rev. Lett., 48, 1175, 1982.

Treumann, R., LaBelle, J., and Bauer, T.: Diffusion Processes: An Observational Perspective, in: Physics of the Magnetopause, Geophys. Mon. 90, AGU, (Eds) Song, P., Sonnerup, B., and Thomsen, M., p. 331, 1995.

Tsurutani, B., Arballo, J., Lakhina, G., Ho, C., Buti, B., Pickett, J., and Gurnett, D.: Plasma waves in the dayside polar cap boundary layer: Bipolar and monopolar eletric pulses and whistler mode waves, Geophys. Res. Lett., 25, 4117, 1998. 